\title{
Double layer onset inside the near-electrode sheath of a RF capacitive discharge in oxygen
}

\author{
V.A. Lisovskiy*, V.D. Yegorenkov \\ Kharkov National University, Svobody Sq. 4, 61077, Kharkov, Ukraine
}

Received 30 March 2005; received in revised form 24 July 2005

\begin{abstract}
This paper reports the axial profiles of the electron temperature $T_{\mathrm{e}}$ in the RF capacitive discharge in oxygen recorded with a probe technique. We observed the $T_{\mathrm{e}}$ peaks near the boundaries of the sheaths as well as inside the near-electrode sheath. The $T_{\mathrm{e}}$ peak inside the sheath is, probably, due to the formation of the double layer at the anode phase of this near-electrode sheath. The assumption of a double layer formation is also supported by the photos of the sheath glow making evident the bright region inside the sheath. The results of our measurements agree with our fluid simulation satisfactorily.
\end{abstract}

(C) 2005 Elsevier Ltd. All rights reserved.

Keywords: Radio-frequency capacitive discharge; Low pressure; Double layer; Probe; Negative ions

\section{Introduction}

Radio frequency capacitive discharge in oxygen is widely applied for etching and modifying the surfaces of polymer materials [1-3], cleaning and oxidizing the surfaces of semiconductor materials $[4,5]$. A large number of experimental and theoretical papers studying the characteristics of this type of the discharge were published (see, e.g., [6-17]). Let us present a brief review of main results obtained with the RF discharge in oxygen.

\footnotetext{
${ }^{*}$ Corresponding author. Tel.: +380573352550 fax: + 380573352530 .

E-mail address: lisovskiy@yahoo.com (V.A. Lisovskiy).
}

Van Roosmalen et al. [6] carried out experiments in $13.56 \mathrm{MHz}$ oxygen discharge at $6.67-53.3 \mathrm{~Pa}$ and $50-800 \mathrm{~W}$. They attempted to reconcile their experimental results with various theoretical models and found that more than half the dissipated power remains physically unaccounted for. Jurgensen [7] proposed a model of the near-electrode sheath of the RF discharge in oxygen. The effect of sheath collision processes on the energy and directionality of surface bombardment in reactive ion etching was modelled. Equations were derived for the average energy of ions and neutrals and the ion energy distribution function. Misium [8] presented a macroscopic model of the planeparallel RF discharge in oxygen predicting the 
plasma characteristics against the parameters under control (gas pressure, power, inter-electrode gap), as well as made a number of recordings of $\mathrm{RF}$ voltage, current and power in a symmetric discharge. Sommerer and Kushner [9] developed a hybrid Monte Carlo fluid simulation which was applied to a variety of gas mixtures of interest to materials processing (including oxygen and their mixtures with helium, nitrogen and $\mathrm{CF}_{4}$ ). Lichtenberg et al. [10] developed a macroscopic analytic model for three-component electronegative oxygen plasma. A positive ion ambipolar diffusion equation was found. Stoffels et al. [11] measured electron and negative ion densities in the 13.56 MHz RF discharge by means of a microwave resonance technique in combination with photo detachment. They developed a kinetic model, incorporating volume and wall reactions of ions as well as neutral species. Vender et al. [12] measured the negative ion density profile in lowpressure oxygen RF plasma by a photo detachment technique and considered the discharge kinetics and the charged particle balance. Feoktistov et al. [13] developed a self-consistent numerical model for an RF discharge in oxygen and calculated the energy distributions of both charged and neutral plasma components using a Monte Carlo method. Aoyagi et al. [14] observed the intense OI emission just in front of the instantaneous cathode in $\mathrm{O}_{2}$ gas discharge at $1 \mathrm{MHz}$, which is considered to be due to the excitation caused by the recombination collisions. Shibata et al. [15] performed numerical analysis of oxygen RF discharge between parallel plates with laser irradiation parallel to the electrode using the relaxation continuum model. They also presented [16] numerical evidence regarding a new type of self-sustaining mechanism for RF oxygen plasma when new electrons are generated in the bulk plasma by the detachment reaction from negative ions with high density under the condition of a relatively high bulk field. Katch et al. [17] found that the plasma of a $13.56 \mathrm{MHz}$ capacitively coupled oxygen discharge of the GEC reference cell exhibits fluctuations of the plasma potential and light emission in the kilohertz range. They explained this behaviour in terms of an attachment-induced ionisation instability in connection with a sufficiently high fraction of negative ions. Wang et al. [18] used the fundamental kinetic equation to derive a space- and time-averaged kinetic equation to analytically calculate electron energy probability function for the RF discharge in oxygen. Gupta and Govinda Raju [19] investigated the dissipated power in an RF discharge in oxygen and found that the ohmic heating in the pre-sheath region and not stochastic heating in the sheath region may be the predominant mechanism. Berezhnoj et al. [20] measured the charged species profiles in oxygen plasma of asymmetrically driven capacitively coupled RF discharge by Langmuir probe technique in the range of pressures from 2.67 to $26.7 \mathrm{~Pa}$ and for inter-electrode distances of $2-10 \mathrm{~cm}$. Kaga et al. [21] obtained the spatial profiles of negatively charged particles in RF oxygen discharge over the pressure range from 13.3 to $66.7 \mathrm{~Pa}$ using a Langmuir probe. Bryant et al. [22] reported on actively compensated Langmuir probe measurements of low-pressure oxygen plasmas in an RF discharge at $13.56 \mathrm{MHz}$. They also presented the theory for both spherical and cylindrical probes in electronegative plasmas. Morscheidt et al. [23] performed comparison between fluid and PIC/MCC numerical models for the simulation of the coupled phenomena of discharge dynamics and chemistry in RF oxygen discharges. Lisovskiy and Yegorenkov [24] reported the recorded current-voltage characteristics of the capacitive RF discharge in oxygen, the extinction curve of the discharge and the $\alpha-\gamma$ transition curve (the curve depicting the transition of the discharge from a weak-current to a strongcurrent mode), electron temperature and axial profiles of the plasma density. It was shown that in a certain range of oxygen pressures the $\alpha-\gamma$ transition was accompanied by low-frequency oscillations of the plasma potential in the kilohertz frequency range. It was obtained that the region of existence of the weak-current regime of the RF capacitive discharge was limited not only from the medium pressure side, but also from the low pressure one.

Makabe et al. theoretically predicted the existence of a double layer at the anode phase of the $\mathrm{RF}$ capacitive discharge in electronegative gases, i.e., in $\mathrm{SF}_{6}$ [25,26], $\mathrm{O}_{2}$ [27], $\mathrm{SiH}_{4}$ [28] and $\mathrm{CF}_{4}$ [29]. 
Formation of this double layer is an important mechanism of the RF discharge sustainment.

The present paper reports on the axial profiles of the electron temperature in the $\mathrm{RF}$ capacitive discharge in low-pressure oxygen recorded with a probe technique. In contrast to the electron temperature profiles in electropositive gases possessing a sharp peak near the boundary of the near-electrode sheath, the second peak located inside the sheath is observed in the electron temperature profile in oxygen. Using the SigloRF fluid code we demonstrated that such second peak appears due to the formation of the double layer at the anode phase of the near-electrode sheath.

\section{Experimental conditions}

Fig. 1 depicts the scheme of the experimental device used for registering the RF discharge characteristics. The section of a fused silica cylindrical tube (not shown in Fig. 1) with inner diameter of $100 \mathrm{~mm}$ vacuum sealed on both ends with stainless-steel planar round electrodes (1) served as a discharge chamber. One of the electrodes of the discharge chamber was grounded. The gas supply system (2) delivered oxygen into the chamber through small orifices in a grounded electrode. The chamber was pumped through the orifices (3) in the same electrode. We used a thermoelectric gauge (4) for registering gas pressure from $0.1 \mathrm{~Pa}$ to the atmospheric one. The pumping was accomplished with pre-vacuum and

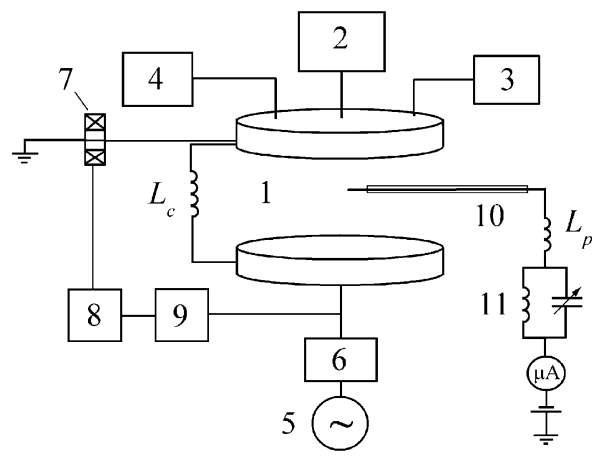

Fig. 1. Experimental set-up. turbo molecular pumps permitting to achieve the limiting pressure of $2.67 \times 10^{-4} \mathrm{~Pa}$. The generator (5) delivered the RF voltage to another electrode via the matching box (6). The choke of $L_{\mathrm{c}}=4 \mathrm{mH}$ inductance switched across the electrodes outside the chamber removed the self-bias voltage.

The experiments were performed within the gas pressure range $p=13.3-66.7 \mathrm{~Pa}$ in the range of $\mathrm{RF}$ voltage amplitudes $U_{\mathrm{RF}} \leqslant 700 \mathrm{~V}$ and the RF field frequency of $f=13.56 \mathrm{MHz}$. The distance between the electrodes was $L=3.3 \mathrm{~cm}$. The amplitude of the RF current $I_{\mathrm{RF}}$ was measured with a Rogowski coil (7) located on the bus, which grounded one of the electrodes. The signal from the Rogowski coil was fed to the FK2-12 device (8). The signal from the capacitive divider (9) connected to the RF electrode was fed to another input of the FK2-12 device. The device for measuring the phase difference FK2-12 is capable of registering the amplitude of the RF signal having the frequency in the range of $f_{1}=$ $1 \mathrm{MHz}-1 \mathrm{GHz}$ and phase shift $\varphi$ between two signals (in our case between RF voltage and RF current). In the absence of the discharge the registered phase shift value is $\varphi=90^{\circ}=\pi / 2$, i.e., we registered only the displacement current. On igniting the RF discharge the active RF current appeared, the phase shift angle $\varphi$ becoming less than $\pi / 2$. The accuracy of measuring the phase shift angle with FK2-12 was 0.1 degree.

Electron temperature was registered with a single cylinder nichrome probe (10) $(5.5 \mathrm{~mm}$ in length and $0.18 \mathrm{~mm}$ in diameter). Cylinder probe was located such that its axis was parallel to the electrode plane. With the probe diameter of $0.18 \mathrm{~mm}$ one can reliably register the profiles of plasma parameters with the step of $0.5-1 \mathrm{~mm}$. The probe length $(5.5 \mathrm{~mm})$ was chosen such that the probe current registered was sufficiently large. A shorter probe (of the length, say, $2 \mathrm{~mm}$ ) would certainly disturb plasma less, but then one would be obliged to account for a complicated shape of the near-probe sheath around the probe's end. A relatively large probe diameter $(0.18 \mathrm{~mm})$ was chosen on the ground of the conclusions of paper [30], where they say that for smaller probes the ion current starts to be affected by the orbital motion of the ions. We also took into account that with 
the dimensions chosen the probe kept its shape even at high temperature (on measuring a high electron current at the positive probe potential with respect to the plasma potential) in the broad range of discharge parameters. The probe with a lesser radius usually is distorted having been heated with the electron current.

We employed the resonance filter (11) consisting of a high-frequency choke and a variable capacitor tuned to the frequency $f$ of the RF generator to prevent the RF current from penetrating the probe circuit. We also included an additional choke of $L_{\mathrm{p}}=10 \mathrm{mH}$ inductance to suppress the main frequency signal and its harmonics in the probe circuit. Electron temperature $T_{\mathrm{e}}$ was determined from the linear sections of the probe current-voltage characteristics drawn to a semi-logarithmic scale, as well as from the second derivative of the probe current with respect to the dc voltage on the probe. The electron temperature $T_{\mathrm{e}}$ values measured with both techniques differed not more than $10-20 \%$. The presence of negative ions does not affect these techniques of measuring the electron temperature [31,32], what ensures the correct determination of $T_{\mathrm{e}}$. To measure the second derivative $\mathrm{d}^{2} I_{\mathrm{pr}} / \mathrm{d} U_{\mathrm{pr}}^{2}$, the second harmonic technique was applied, i.e., the probe current was modulated with a low-frequency voltage (with the frequency $f_{\text {If }} \approx 1-3 \mathrm{kHz}$ ), but the signal was registered at the frequency $2 f_{\text {lf }}$. The set-up for measuring $\mathrm{d}^{2} I_{\mathrm{pr}} / \mathrm{d} U_{\mathrm{pr}}^{2}$ was described in paper [33]. Regretfully, this set-up did not permit to make correct measurements of the second derivative $\mathrm{d}^{2} I_{\mathrm{pr}} / \mathrm{d} U_{\mathrm{pr}}^{2}$ near the plasma potential. Therefore we could not employ the technique for measuring the temperature $T_{n}$ and density $n_{n}$ of negative ions suggested in [31,32].

\section{Experimental results}

Consider the electron energy probability functions (EEPF) recorded with our single Langmuir probe in the near-electrode sheath and at the discharge centre (Fig. 2). The EEPF, drawn to a semi logarithmic scale, possesses at the discharge centre an approximately Maxwell shape with a well-expressed linear section (which we used for
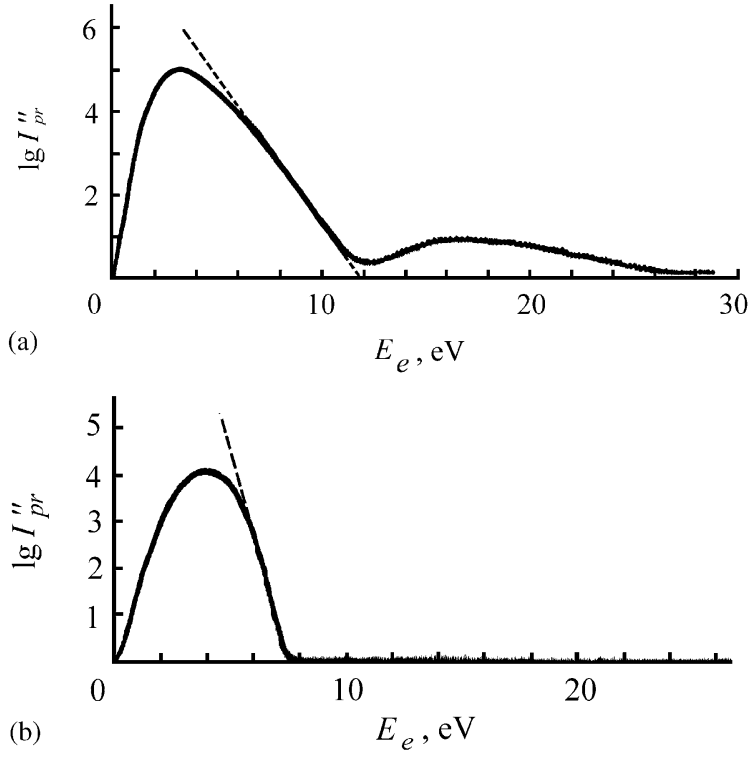

Fig. 2. Electron energy probability functions in the nearelectrode sheath $(x=3 \mathrm{~mm})$ (a) and at the discharge centre (b).

determining the electron temperature) and does not have a "tail" due to high-energy electrons. The EEPF recorded in the near-electrode sheath possesses two electron populations, namely, a Maxwell one and a high-energy one. In this case the EEPF "tail" consists of secondary electrons hit from the electrode surface by ions and photons and accelerated in the strong electric field at the cathode phase of the sheath. Plasma electrons can also acquire high energy under stochastic heating colliding with the oscillating boundary of the sheath (at the cathode phase of the sheath). A population of low-energy electrons collected by the probe in the sheath, probably, relates to the anode phase of the sheath when the boundary of the quasi-neutral plasma is moving to the electrode, the sheath is filled up with electrons and collapses. In a number of cases this section of EEPF contains a linear section; therefore we can evaluate the electron temperature from its tilt with respect to the abscissa axis (assuming the low energy electrons to possess a Maxwell distribution). The electron temperature thus determined turns up to be remarkably higher than one at the centre of the discharge gap in agreement with the results of papers [34-37]. 


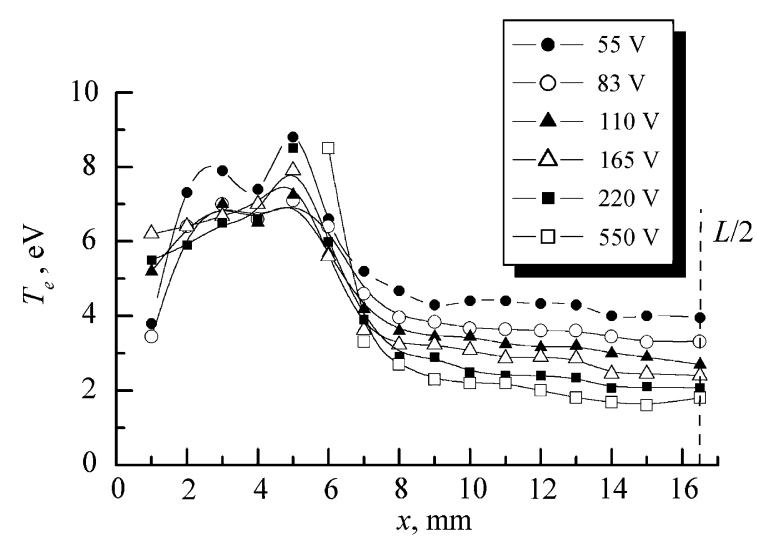

Fig. 3. Axial profiles of the electron temperature at $p=13.3 \mathrm{~Pa}$ for different RF voltage values.

Fig. 3 shows the axial profiles of the electron temperature (from the electrode surface to the discharge centre) we recorded at the oxygen pressure of $13.3 \mathrm{~Pa}$ and various values of the RF voltage. The measured thickness of the nearelectrode sheath amounted about $5 \mathrm{~mm}$. The profiles of the electron temperature in the figure clearly possess two peaks. One of them is located near the boundary of the sheath whereas another one turned up to be inside the sheath at the distance of about $3 \mathrm{~mm}$ from the electrode surface. Regarding the observation of two peaks in the electron temperature profile we can say that: (1) at voltages above $55 \mathrm{~V}$ the registration of this pattern might require increased spatial resolution, and (2) with the voltage increasing there is a clear tendency of smoothing. The naked eye observed a luminescent layer much brighter than the adjacent regions of the sheath (excluding, of course, a boundary of the near-electrode sheath with a brighter glow). Consequently, a region exists inside the near-electrode sheath in which electrons can gain energy and perform stronger excitations and ionisations of gas molecules.

We employed the fluid Siglo-RF code, freely downloaded from the Kinema site (http://www. kinema.com/), for better understanding of the processes in the RF capacitive discharge in oxygen. This code permits to calculate the axial profile of the average energy of electrons $\left\langle\varepsilon_{\mathrm{e}}\right\rangle$. Siglo-RF is a three-electron-moment, two-ion-

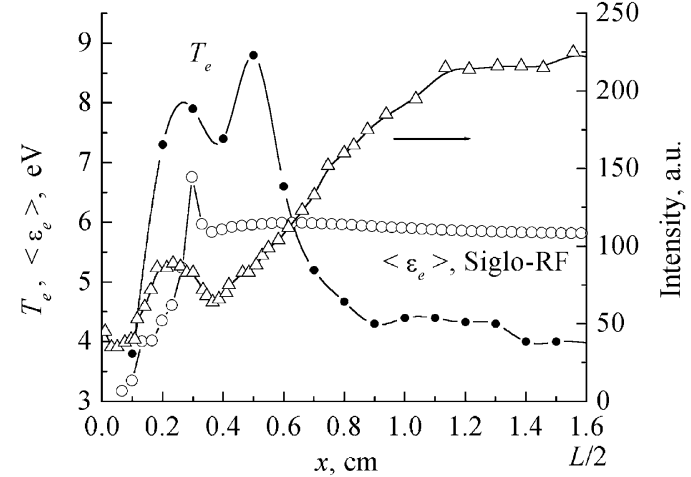

Fig. 4. Axial profiles of the electron temperature $T_{\mathrm{e}}$ (experiment) and of the average electron energy $\left\langle\varepsilon_{\mathrm{e}}\right\rangle$ (calculation with the Siglo-RF code) at $p=13.3 \mathrm{~Pa}, U_{\mathrm{RF}}=55 \mathrm{~V}$.

moment fluid model. The electrons are described by the continuity, momentum and energy equations and the ions by the continuity and momentum equations. Positive and negative ions are both considered. These fluid equations are coupled to Poisson's equation for the electric potential.

Fig. 4 depicts one example of such calculations for oxygen pressure of $13.3 \mathrm{~Pa}$ and $\mathrm{RF}$ voltage of $55 \mathrm{~V}$. The calculated $\left\langle\varepsilon_{\mathrm{e}}\right\rangle$ profile possesses two peaks (near the boundary of and inside the nearelectrode sheath) similar to the electron temperature profile recorded in experiments. The locations of the $T_{\mathrm{e}}$ and $\left\langle\varepsilon_{\mathrm{e}}\right\rangle$ peaks inside the near-electrode sheath also coincide. The peak (located near the boundary of the sheath) obtained with the Siglo$\mathrm{RF}$ code is very weakly expressed, because the Siglo-RF code is a fluid code. The authors of paper [38] compared the axial profiles of plasma parameters obtained via a fluid model and a particle-incell/Monte Carlo (PIC/MC) model of a onedimensional, capacitively coupled RF discharge. They found that in the sheath regions, the PIC/ MC model predicts a significant rise in the mean electron energy which is not observed in the fluid model. The sharp rise in mean electron energy in the sheath predicted by the PIC/MC model is due to a continual presence of the most energetic electrons in the sheath regions over the RF period. The high-energy electrons are weighted more heavily in the calculation of the mean energy because there are fewer low-energy electrons present in the sheath at all times in the RF cycle, 


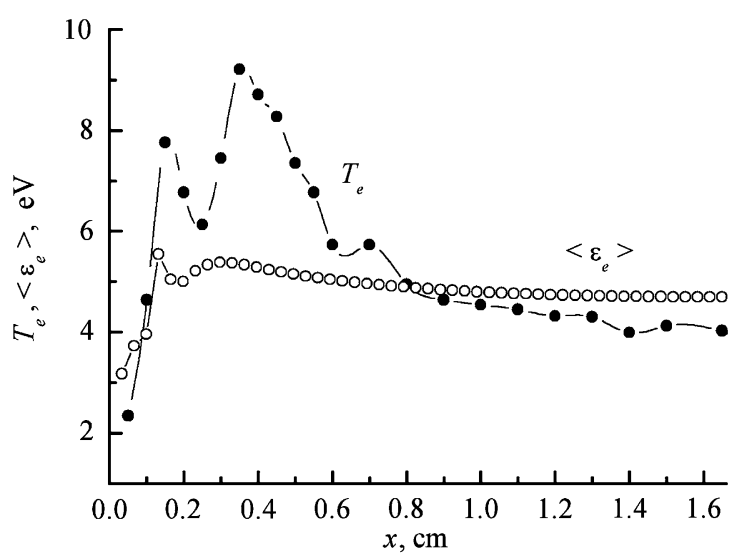

Fig. 5. Axial profiles of the electron temperature $T_{\mathrm{e}}$ (experiment) and of the average electron energy $\left\langle\varepsilon_{\mathrm{e}}\right\rangle$ (calculation with the Siglo-RF code) at $p=66.7 \mathrm{~Pa}, U_{\mathrm{RF}}=130 \mathrm{~V}$.

but particularly when the sheaths are in their anodic phase.

Fig. 5 shows similar $T_{\mathrm{e}}$ and $\left\langle\varepsilon_{\mathrm{e}}\right\rangle$ profiles for oxygen pressure of $66.7 \mathrm{~Pa}$. Under these conditions the near-electrode sheath was about $3.5 \mathrm{~mm}$ thick, and the second peak of the $T_{\mathrm{e}}$ profile (and the glowing layer) was at the distance of about $2 \mathrm{~mm}$ from the electrode.

As is known [11,12], the RF discharge in oxygen contains a considerable number of negative ions. In order to prove that the $T_{\mathrm{e}}$ peak inside the sheath turns up just in electronegative gases, we performed additional measurements and calculations for argon (the profile of electron temperature shown in Fig. 6 for argon was registered in the chamber with a lesser inter-electrode gap $L=22 \mathrm{~mm}$ ). It follows from Fig. 6 that the $T_{\mathrm{e}}$ and $\left\langle\varepsilon_{\mathrm{e}}\right\rangle$ profiles in the RF discharge in argon possess only a single peak located near the boundary of the nearelectrode sheath. On approaching the electrode surface the $T_{\mathrm{e}}$ and $\left\langle\varepsilon_{\mathrm{e}}\right\rangle$ decrease uniformly. We observed no additional glowing regions inside the sheath in argon. The experimental data we obtained for $T_{\mathrm{e}}$ are in good agreement with the calculations from [37]. At the same time we observed an additional brightly shining layer inside the nearelectrode sheath of the RF discharge in electronegative gases $\mathrm{SF}_{6}$ and $\mathrm{CF}_{4}$. However, the probe measurements in these gases are impeded, because the surface of the probe is quickly coated with a

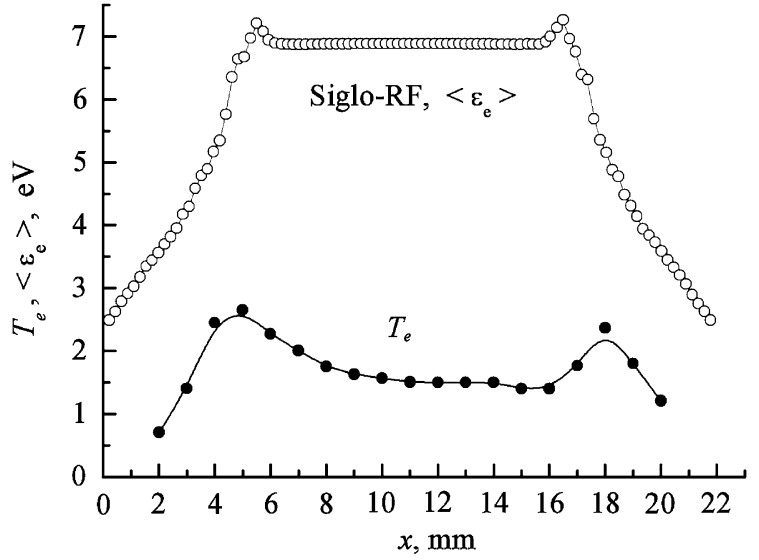

Fig. 6. Axial profiles of the electron temperature $T_{\mathrm{e}}$ (experiment) and of the average electron energy $\left\langle\varepsilon_{\mathrm{e}}\right\rangle$ (calculation with the Siglo-RF code) in argon for $L=22 \mathrm{~mm}, p=13.3 \mathrm{~Pa}$, $U_{\mathrm{RF}}=220 \mathrm{~V}$.

sulphur film (in $\mathrm{SF}_{6}$ ) or a polymer film in $\mathrm{CF}_{4}$, which are difficult to remove simply by heating the probe to a high temperature. Therefore we cannot depict here the axial $T_{\mathrm{e}}$ profiles for $\mathrm{SF}_{6}$ and $\mathrm{CF}_{4}$. But the presence of the glowing region in the nearelectrode sheath in electronegative gases like $\mathrm{O}_{2}$, $\mathrm{SF}_{6}$ and $\mathrm{CF}_{4}$ indicates that negative ions play a role in its formation.

One can argue on the validity of Langmuir probes in space-charge regions, in electronegative cases, in presence of anisotropic velocity distributions, etc. but even when the probe does not provide high-accuracy data, it allows at least to detect some basic features. As the naked eyes report of the bright region inside the sheath that means double layer is regarded to be the most reliable evidence. We made photos of the nearelectrode glows and digitized them.

Fig. 7 shows the photos of near-electrode sheaths in RF discharge in oxygen and argon $\left(p=13.3 \mathrm{~Pa}\right.$ and $\left.U_{\mathrm{RF}}=150 \mathrm{~V}\right)$. The photos provide the evidence that a bright luminous layer is observed inside the near-electrode sheath in oxygen whereas it is absent in argon. We digitized these photos, and Fig. 8 depicts in blue colour the output intensity distributions of luminosity in oxygen and argon discharges across the sheath width. A slight increase in the intensity of the glow near the electrode surface is due to the reflection of 

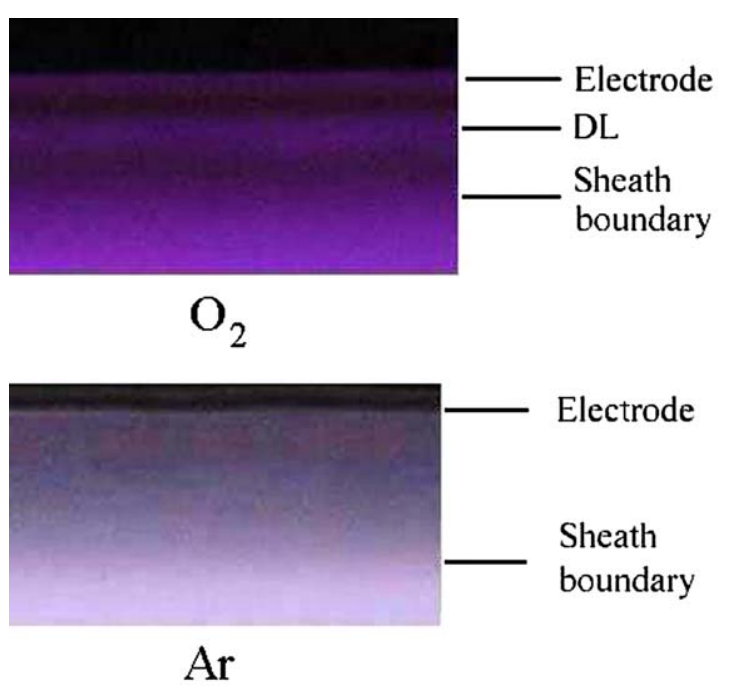

Fig. 7. Photos of near-electrode sheaths in RF discharges in oxygen and argon $\left(p=13.3 \mathrm{~Pa}\right.$ and $\left.U_{\mathrm{RF}}=150 \mathrm{~V}\right)$.

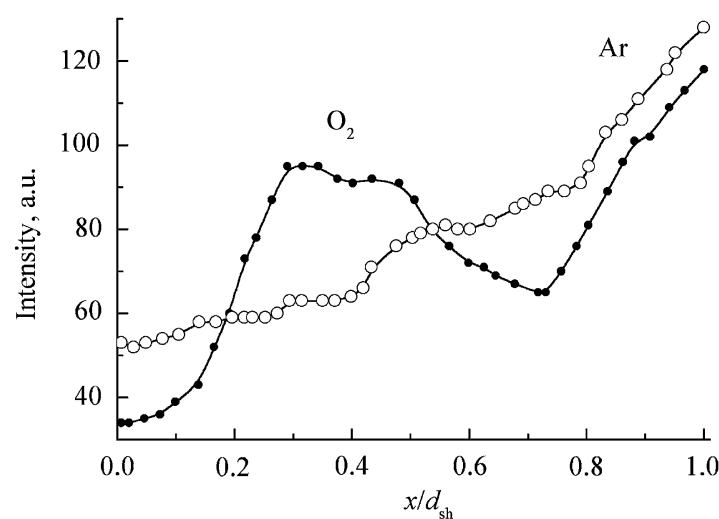

Fig. 8. Intensity distributions of discharge glow in oxygen and argon shown in blue colour across the sheath width, $p=13.3 \mathrm{~Pa}$ and $U_{\mathrm{RF}}=150 \mathrm{~V}$.

light off the electrode. We also presented a similar profile of luminosity of RF discharge in oxygen in Fig. 4. The location of the luminous layer of the electron temperature and average energy peaks inside the near-electrode sheath agree well between themselves. Thus, though the application of the probe technique for measurements inside a nearelectrode sheath might be subjected to equitable criticism, just the probe current-voltage characteristics were the first to indicate the enhanced local heating of electrons inside the near-electrode sheath in oxygen (and its absence in argon).

Now let us consider the time-averaged profiles of the RF potential $U, \mathrm{RF}$ electric field $E$, concentrations of electrons $N_{\mathrm{e}}$, of positive $N_{\mathrm{i}}$ and negative $N_{\mathrm{n}}$ ions calculated with the SigloRF code (Fig. 9). The $U$ and $E$ profiles for oxygen presented in this figure are similar to the profiles for electropositive gases qualitatively [39-41]. The concentration of negative ions $N_{\mathrm{n}}$ in the discharge volume almost coincides with the concentration of positive ions $N_{\mathrm{i}}$ but for the near-electrode sheaths. The negative ions are trapped in the discharge volume being contained in the potential well; therefore, they are almost absent in the nearelectrode sheaths. The $N_{\mathrm{i}}$ and $N_{\mathrm{n}}$ profiles in the quasi-neutral plasma are bell-shaped. The electron concentration $N_{\mathrm{e}}$ in the discharge volume is almost

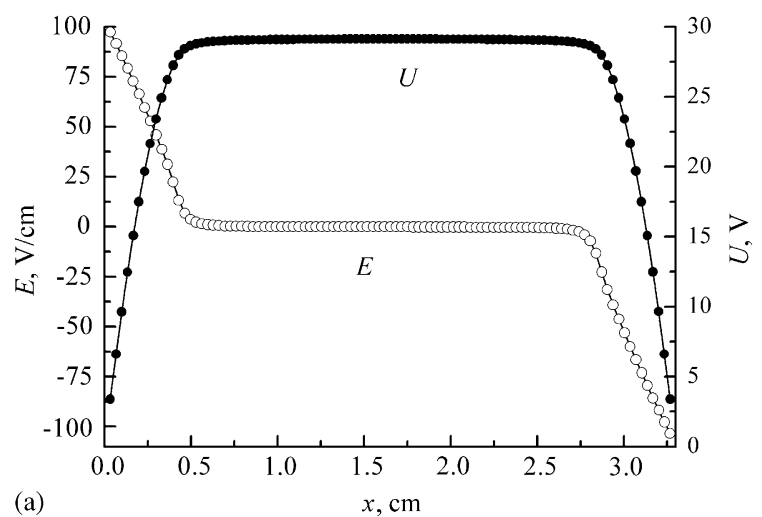

(a)

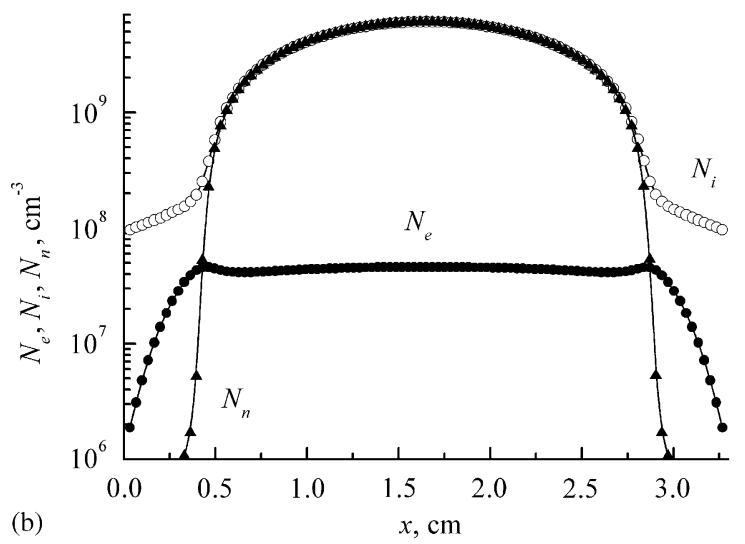

Fig. 9. Time-averaged profiles of RF potential $U$ and RF electric field $E$ (a), concentrations of electrons $N_{\mathrm{e}}$, positive $N_{\mathrm{i}}$ and negative $N_{\mathrm{n}}$ (b) ions at $p=13.3 \mathrm{~Pa}, U_{\mathrm{RF}}=55 \mathrm{~V}$. 
constant; we observe the weakly expressed $N_{\mathrm{e}}$ only inside the near-electrode sheaths.

In Fig. 9 (as well as in Fig. 10) the grounded electrode is to the left $(x=0)$, and the RF electrode is to the right $(x=3.3 \mathrm{~cm})$. Fig. 10 presents the axial profiles for the time moment when the RF electrode possesses the maximum negative potential $U_{\mathrm{el}}=-55 \mathrm{~V}$. The near-electrode sheath near the RF electrode is in the cathode phase; it is almost free of electrons. At the same time the near-electrode sheath near the grounded electrode is in the anode phase and filled with electrons. It is quite clear from Fig. 10 that a double layer is formed in the left sheath, the peculiarities being present in the profiles of the potential $U$ and RF electric field $E$. The profile of the electron concentration $N_{\mathrm{e}}$ possesses a wellexpressed peak inside the near-electrode sheath.
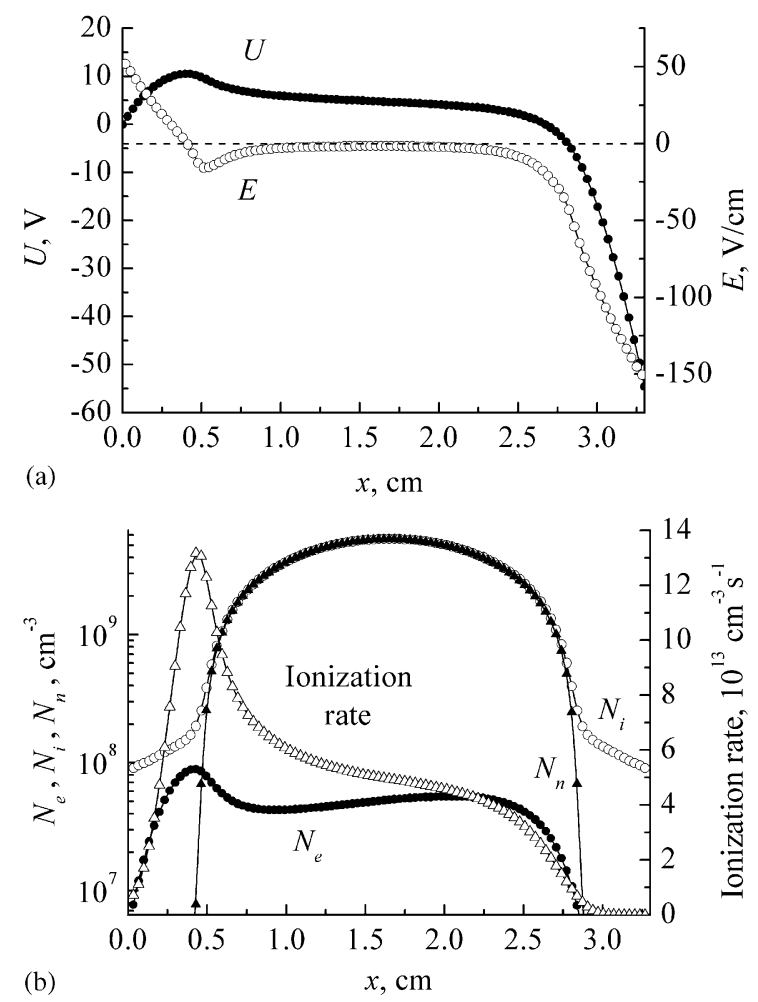

Fig. 10. Axial profiles of RF potential $U$ and RF electric field $E$ (a), concentrations of electrons $N_{\mathrm{e}}$, positive $N_{\mathrm{i}}$ and negative $N_{\mathrm{n}}$ (b) ions as well as of the ionisation rate at $p=13.3 \mathrm{~Pa}, U_{\mathrm{RF}}=$ $55 \mathrm{~V}$ for the time moment when the potential of the RF electrode is equal to $U_{\mathrm{el}}=-55 \mathrm{~V}$.
This is caused by the increased ionisation rate in the region of existence of the double layer which 2-3 times exceeds one at the centre of the discharge gap in the quasi-neutral plasma. The authors of papers [25-29,36,37,39,40,42] obtained similar $U$, $E$ and $N_{\mathrm{e}}$ profiles for the anode phase of the nearelectrode sheath of the RF discharge in electronegative gases. Perhaps it is the onset of the double layer at the anode phase that leads to the local increase of the electron temperature inside the near-electrode sheath we discovered in the experiment.

In the RF discharge the numbers of electrons and positive ions hitting the electrode surface during the period $T$ of the RF field must be equal. Positive ions come to the electrode surface in the form of the almost constant current $I_{i}$, modulated with the RF field frequency. Electrons can approach the electrode surface only at the anode phase of the sheath, the electron current $I_{\mathrm{e}}$ to the electrode having the pulsed form (Fig. 11). The areas $\int_{0}^{T} I_{i}(t) \mathrm{d} t$ and $\int_{0}^{T}\left|I_{\mathrm{e}}(t)\right| \mathrm{d} t$ must be equal. In the RF discharge in electropositive gases this condition is easily met, because in plasma $N_{\mathrm{i}} \approx N_{\mathrm{e}}$. However, in electronegative gases in plasma we have $N_{\mathrm{e}} \ll N_{\mathrm{i}}$. For example, we obtain from Fig. 9 at the centre of the discharge $N_{\mathrm{e}} \approx 4.62 \times 10^{7} \mathrm{~cm}^{-3}, N_{\mathrm{i}} \approx 6.11 \times 10^{9} \mathrm{~cm}^{-3}$, $N_{\mathrm{n}} \approx 6.064 \times 10^{9} \mathrm{~cm}^{-3}$, i.e., $N_{\mathrm{e}} / N_{\mathrm{n}} \approx 7.6 \times 10^{-3}$. Consequently, the majority of electrons in the discharge volume are attached to oxygen molecules

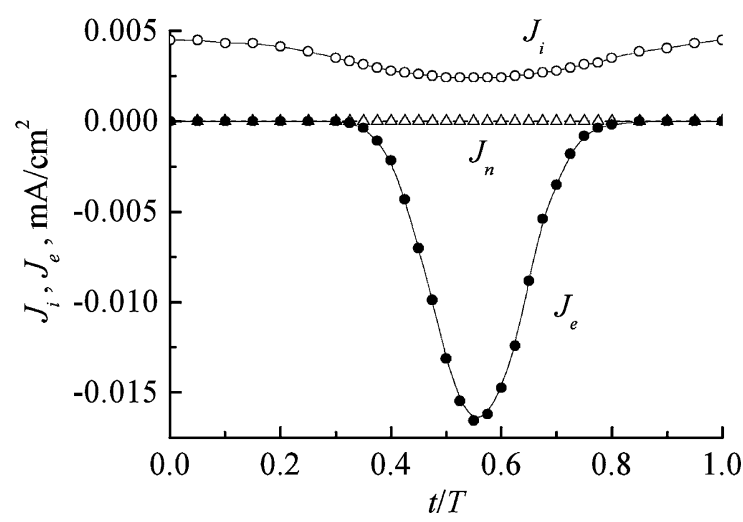

Fig. 11. Current densities of electrons $J_{\mathrm{e}}$, positive $J_{\mathrm{i}}$ and negative $J_{\mathrm{n}}$ ions hitting the grounded (left) electrode during the period of the RF field $T, p=13.3 \mathrm{~Pa}, U_{\mathrm{RF}}=55 \mathrm{~V}$. 
forming negative ions, and they cannot get to the electrode surface and compensate the positive charge. Therefore, at the anode phase of the sheath, when the remaining free electrons are moving to the electrode, a double layer appears in the nearelectrode sheath. It is the double layer that heats electrons locally, increasing the ionisation rate abruptly and generating the required additional flow of electrons to the electrode. Only in this case the number of positive and negative charges hitting the electrode surface during the RF field period can be equal.

As is known [42], two conditions should be met for a double layer to be formed in an electronegative gas. First, the concentration of negative ions should be sufficiently high for the negative ions to carry a noticeable part of the discharge current:

$N_{-} \geqslant \frac{N_{\mathrm{e}} \mu_{\mathrm{e}}}{\mu_{\mathrm{n}}}$,

where $\mu_{\mathrm{e}}$ and $\mu_{\mathrm{n}}$ are the mobilities of electrons and negative ions, respectively. Second, the ratio of electron concentration to that of negative ions in the sheath should exceed one in plasma:

$\left(\frac{N_{\mathrm{e}}}{N_{\mathrm{n}}}\right)_{\text {sheath }}>\left(\frac{N_{\mathrm{e}}}{N_{\mathrm{n}}}\right)_{\text {plasma }}$.

Let us check the validity of these criteria under our conditions. In the RF discharge in oxygen the $\mathrm{O}^{-}$ions dominate whereas $\mathrm{O}_{2}^{-}$ion content amounts to not more than $10-20 \%$ [27]. Mobilities of these ions in oxygen are equal to $1.28 \times 10^{4}$ Torr $\mathrm{cm}^{-2} \mathrm{~V}^{-1} \mathrm{~s}^{-1}$ and $8.63 \times$ $10^{3}$ Torr $\mathrm{cm}^{-2} \mathrm{~V}^{-1} \mathrm{~s}^{-1}$, respectively [43], and we assume the electron mobility to be equal to $3.63 \times 10^{5}$ Torr $\mathrm{cm}^{-2} \mathrm{~V}^{-1} \mathrm{~s}^{-1}$ [44]. As the Siglo$\mathrm{RF}$ code does not permit to obtain the concentrations of negative ions $\mathrm{O}^{-}$and $\mathrm{O}_{2}^{-}$separately, we will perform evaluations for two cases, namely, we will assume the discharge to contain a single species of negative ions. Then

$$
\begin{aligned}
N_{\mathrm{n}} & =6.064 \times 10^{9} \mathrm{~cm}^{-3} \\
& >N_{\mathrm{e}} \frac{\mu_{\mathrm{e}}}{\mu_{\mathrm{n}}}=1.31 \times 10^{9} \mathrm{~cm}^{-3} \text { for } \mathrm{O}^{-} \\
& =1.94 \times 10^{9} \mathrm{~cm}^{-3} \text { for } \mathrm{O}_{2}^{-}
\end{aligned}
$$

Consequently, the criterion (1) holds. Besides, it follows from the results of the Siglo-RF code that the RF current of negative ions at the discharge centre constitutes almost one third of the electron current. Above we wrote that $N_{\mathrm{e}} / N_{\mathrm{n}} \approx 7.6 \times 10^{-3}$ at the discharge centre. Near the boundary of the near-electrode sheath we have $N_{\mathrm{e}} \approx N_{\mathrm{n}}$, and within it $N_{\mathrm{e}} \gg N_{\mathrm{n}}$, i.e., the criterion (2) also holds. Therefore, the favourable conditions exist in our discharge for a double layer to appear in the anode phase of the near-electrode sheath.

\section{Conclusions}

We recorded the axial profiles of the electron temperature $T_{\mathrm{e}}$ in the RF discharge in oxygen with a single cylindrical Langmuir probe. The $T_{\mathrm{e}}$ profiles possess peaks at the boundary of the sheaths as well as inside the near-electrode sheath. An additional glow is observed in the same region of the nearelectrode sheath indicating the enhanced ionisation and excitation of gas molecules via electron impact. The appearance of the $T_{\mathrm{e}}$ peak and the glow inside the sheath are associated, probably, with a double layer formation in the anode phase of the nearelectrode sheath. The existence of this double layer is supported by the predictions of the fluid Siglo-RF code. We also show that the criteria for a double layer to appear in an electronegative gas hold under conditions of our experiments.

\section{Appendix A. Supplementary data}

Supplementary data associated with this article can be found in the online version at doi:10.1016/ j.vacuum.2005.07.038.

\section{References}

[1] Baggerman JAG, Visser RJ, Collart EJH. J Appl Phys 1994:75:758.

[2] Meichsner J, Zeuner M, Krames B, Nitschke M, Rochotzki R, Barucki K. Surface and Coatings Technology 1998;98:1565.

[3] Cepeda-Jimenez CM, Torregrosa-Macia R, Martin-Martinez JM. Surf Coat Technol 2003;174-175:94. 
[4] Nakano J, Suzuki M. Vacuum 1986;36:85.

[5] Petasch W, Kegel B, Schmid H, Lendenmann K, Keller HU. Surf Coat Technol 1997;97:176.

[6] Van Roosmalen AJ, van den Hoek WGM, Kalter H. J Appl Phys 1984;58:653.

[7] Jurgensen CW. J Appl Phys 1988;64:590.

[8] Misium GR. J Vac Sci Technol A 1990;8:1642.

[9] Sommerer TJ, Kushner MJ. J Appl Phys 1992;71:1654.

[10] Lichtenberg AJ, Vahedi V, Lieberman MA, Rognlien T. J Appl Phys 1994;75:2339.

[11] Stoffels E, Stoffels WW, Vender D, Kando M, Kroesen GMW, de Hoog FJ. Phys Rev E 1995;51:2425.

[12] Vender D, Stoffels WW, Stoffels E, Kroesen GMW, de Hoog FJ. Phys Rev E 1995;51:2436.

[13] Feoktistov VA, Mukhovatova AV, Popov AM, Rahimova TV. J Phys D 1995;28:1346.

[14] Aoyagi K, Ishikawa I, Saito Y, Suganomata S. Jpn J Appl Phys 1996;35:6248.

[15] Shibata M, Nakano N, Makabe S. Jpn J Appl Phys 1997;36:5290.

[16] Shibata M, Nakano N, Makabe T. J Phys D 1998;37:4182.

[17] Katch H- M, Doehlich A, Kawetzki T, Quandt E, Dobele H-F. Appl Phys Lett 1999;75:2023.

[18] Wang Z, Lichtenberg AJ, Cohen RH. Plasma Sources Sci Technol 1999;8:151.

[19] Gupta N, Govinda Raju GR. J Phys D 2000;33:2949.

[20] Berezhnoj SV, Shin CB, Buddemeier U, Kaganovich I. Appl Phys Lett 2000;77:800.

[21] Kaga K, Kimura T, Ohe K. J Phys D 1997;30:1219.

[22] Bryant P, Dyson A, Allen JE. J Phys D 2001;34:95.

[23] Morscheidt W, Longo S, Hassouni K, Arefi F. In: Proceedings of XXV International Conference on Phenomena in Ionized Gases, 17-22 July 2001, vol. 2, Nagoya, Japan, p.229.
[24] Lisovskiy VA, Yegorenkov VD. Vacuum 2004;74:19.

[25] Nakano N, Shimura N, Petrovic ZL, Makabe T. Phys Rev E 1994;49:4455.

[26] Nakano N, Makabe T. J Phys D 1995;28:31.

[27] Shibata M, Nakano N, Makabe T. J Appl Phys 1995;77:6181.

[28] Makabe T, Tochikubo F, Nishimura M. Phys Rev A 1990;42:3674.

[29] Segawa S, Kurihara M, Nakano N, Makabe T. Jpn J Appl Phys 1999;38B:4416.

[30] Bryant P, Dyson A, Allen JE. J Phys D 2001;34:95.

[31] Amemiya H. J Phys D 1990;23:999.

[32] Amemiya H. Vacuum 2000;58:100.

[33] Dudin SV. Pribory i Tekhnika Experimenta 1994;4:78 [In Russian].

[34] Kushner MJ. J Appl Phys 1983;54:4958.

[35] Sommerer TJ, Hitchon WNG, Harvey REP, Lawler JE. Phys Rev A 1991;43:4452.

[36] Sommerer TJ, Kushner MJ. J Appl Phys 1992;71:1654.

[37] Lymberopoulos DP, Economou DJ. J Phys D 1995;28:727.

[38] Nitschke TE, Graves DB. J Appl Phys 1994;76:5646.

[39] Boeuf JP, Belenguer Ph. In: Capitelli M, Bardsley JN, editors. Nonequlibrium processes in partially ionized gases. New York: Plenum Press; 1990.

[40] Pitchford LC, Belenguer PH, Boeuf JP. In: Ferreira CM, Moisan M, editors. Microwave discharges: fundamentals and applications. New York: Plenum Press; 1993.

[41] Raizer YP, Shneider MN, Yatsenko NA. Radio-frequency capacitive discharges. Boca Raton, FL: CRC Press; 1995.

[42] Gottscho RA. Phys Rev A 1987;36:2233.

[43] McDaniel EW, Mason EA. The mobility and diffusion of ions in gases. New York: Wiley; 1973.

[44] Jeon B-H, Nakamura Y. J Phys D 1998;31:2145. 